\title{
New Insights in Physical Therapy and Rehabilitation in Psoriatic Arthritis: A Review
}

Fabio Massimo Perrotta $\cdot$ Silvia Scriffignano · Devis Benfaremo •

Mario Ronga · Michele Maria Luchetti · Ennio Lubrano (D)

Received: December 24, 2020 / Accepted: March 3, 2021 / Published online: March 12, 2021

(c) The Author(s) 2021

\begin{abstract}
Psoriatic arthritis (PsA) is a complex, multiform and chronic inflammatory disease characterized by the association of psoriasis and arthritis with other musculoskeletal and extra-articular manifestations. The treatment of PsA is rapidly evolving due to the introduction of new biologic and small-molecule drugs, and the aim of treatment is to induce a condition of remission or low disease activity in all disease domains. However, unmet treatment needs still persist for those patients with impaired function, reduced quality of life or comorbidities. In this context, physical therapy and rehabilitation could provide additional benefits by reducing disease
\end{abstract}

F. M. Perrotta $\cdot$ S. Scriffignano $\cdot$ E. Lubrano $(\bowtie)$ Academic Rheumatology Unit, Dipartimento di Medicina e Scienze della Salute "Vincenzo Tiberio", Università degli Studi del Molise, Campobasso, Italy e-mail: enniolubrano@hotmail.com

D. Benfaremo · M. M. Luchetti

Clinica Medica, Dipartimento Scienze Cliniche e Molecolari, Università Politecnica delle Marche, Ancona, Italy

M. Ronga

Orthopedics and Trauma Operative Unit,

Department of Biomedical and Dental Sciences and

Morpho-Functional Imaging, University Hospital G.

Martino, University of Messina, Messina, Italy activity and improving function. Although a large number of studies have assessed the role of physical therapy and exercise in other forms of chronic inflammatory arthritis, such as axial spondyloarthritis and rheumatoid arthritis, evidence on their effect on persons with PsA is still lacking. However, some studies have reported the potential positive role of physical therapy on the different disease domains of PsA, in helping to improve disease activity, prevent or improve articular impairment, improve pain management and improve quality of life. Here, we review current evidence on physical therapy, exercise and rehabilitation in patients with PsA. In particular, we review the literature focusing on each domain, to provide evidence of efficacy and effectiveness of exercise and rehabilitation on skin, peripheral arthritis, axial involvement, dactylitis, enthesitis and comorbidities.

Keywords: Physiotherapy; Psoriatic arthritis; Rehabilitation; Treatment 


\section{Key Summary Points}

Psoriatic arthritis (PsA) is a complex and multiform disease that leads, if untreated, to a reduction of function and quality of life.

Treatment options in PsA are rapidly evolving; however, unmet clinical needs still persist for patients with inadequate response to pharmacological treatments and those with residual disease activity, impaired function and/or pain.

Recent European League Against Rheumatism (EULAR) recommendations include physical therapy and exercise for patients with inflammatory arthritis and PsA.

However, at the present time there is little evidence on the role of physical therapy and exercise in the treatment of the different aspects of psoritic arthritis, including skin, articular involvement, enthesitis and comorbidities; thus, new studies are needed.

\section{DIGITAL FEATURES}

This article is published with digital features, including a summary slide, to facilitate understanding of the article. To view digital features for this article go to https://doi.org/10.6084/ m9.figshare.14140916.

\section{INTRODUCTION}

Psoriatic arthritis (PsA) is a complex inflammatory disease in which different cutaneous, musculoskeletal, extra-articular manifestations and comorbidities come together to define the clinical phenotype [1]. The hallmark of articular manifestations is the presence of arthritis (both peripheral and axial), dactylitis and enthesitis, with a possible association with extra-articular manifestations and comorbidities, leading the physician to take a "holistic" approach when treating patients [2]. In recent years, PsA treatment has rapidly evolved with the introduction of new biologics and small-molecule drugs, and the aim of treatment is to induce remission or low disease activity in all disease domains [3-6]. Data from clinical trials, real-life experiences and systematic reviews have shown the good efficacy and effectiveness of these new treatment approaches, with the possibility to achieve remission or low disease activity in up to two-thirds of patients with PsA $[7,8]$. However, an unmet treatment need still persists for those patients intolerant or non-responders to pharmacological treatments or those with impaired function, reduced quality of life and/ or comorbidities. For example, in the very recent SELECT-PsA 2 trial, the percentage of enrolled patients who were not responders or intolerant to $\geq 3$ biologics ranged between 11 and $16 \%$, representing a potential disease management issue [9]. Furthermore, the complexity of PsA may lead to the presence of residual disease activity in different domains, and some studies have shown how residual pain, impaired function and decreased quality of life could contribute to disease burden [10].

Pain, function, fatigue and health-related quality of life, together with musculoskeletal and skin disease activity, were ultimately included in the OMERACT-endorsed PsA core domain set, which should be taken as a guidance for all treating physicians [11]. In this context, beyond pharmacological treatment strategy, physical therapy, exercise and rehabilitation may play an important role in the overall management of PsA patients, contributing to reduced disease burden in each domain. However, despite the positive role of rehabilitation and physical therapy programs in the management of various musculo-skeletal diseases, there is curently very little evidence on the efficacy or effectiveness of rehabilitation and physical therapy in PsA. In this context, the aim of this article is to narratively review current evidence on the potential role of physical therapy, exercise and rehabilitation in the management PsA patients in each disease domain. 


\section{METHODS}

We searched PubMed and the Cochrane library for articles and reviews in the English language published between 1 Jan 2000 and 30 October 2020. Search terms were "psoriatic arthritis," "psoriasis," "psoriatic disease," "synovitis," "axial involvement," "dactylitis," "enthesitis," "rehabilitation," "exercise," "physical therapy" and "comorbidities," and the search was limited to human clinical studies, clinical trials, reviews and meta-analyses. We followed previously published guidelines for writing a narrative review [12].

We also added other papers suggested by the co-authors in order to improve the quality of the review. This article is based on previously conducted studies and does not contain any studies with human participants or animals performed by any of the authors.

\section{PHYSICAL THERAPY, EXERCISE AND REHABILITATION IN PSA: SKIN INVOLVEMENT}

Psoriasis is an inflammatory skin disease that presents with itching, red, scaling plaques. Patients with PsA generally have a mild skin involvement, but some patients have severe skin disease, with possible involvement of the scalp, hands/feet and genitals, with an important impact on quality of life.

Some studies have reported that worsening of psoriasis is associated with obesity, drinking, smoking, lack of sleep and a sedentary lifestyle, indicating that lifestyle changes and physical activity may, potentially, improve psoriasis $[13,14]$. Psychological stress has also been shown to be an important role in the natural history of psoriasis and its worsening, but the link between the two conditions remains to be clearly defined [15]. It has also been reported that in $31-88 \%$ of cases, patients reported stress as the trigger factor for their psoriasis onset. In this context, understanding the role of stress and its impact on skin psoriasis could be important in the management of patients with psoriasis. In fact, several controlled studies have demonstrated that relaxation, hypnosis, biofeedback and behavioral and cognitive stress management therapies are effective in people with psoriasis [16].

Obesity is a well-known risk factor for psoriasis, and there is a correlation between body mass index (BMI) and extension of psoriasis [17]. In this context, the control of body weight with physical exercise could be important in the management of skin psoriasis. In one study, the control of body weight was shown to improve overall patient health, to reduce oxidative stressors and to positively impact on the Psoriasis Area and Severity Index (PASI) scores [18].

A recent Cochrane review was performed to investigate the impact of dietary and physical exercise on psoriasis [19]. One trial enrolled 303 obese participants with moderate-to-severe psoriasis who had started a systemic therapy for psoriasis and did not achieve clearance; a combined dietary intervention and exercise program was applied [20]. Other studies involved using continuous health education programs, diet and walking exercises [19]. Overall, these studies showed a beneficial effect of diet and exercise on the reduction of psoriasis as assessed by the PASI, as well as a reduction of the BMI $[19,20]$. Finally, walking exercise combined with continuous health education seem to have beneficial effects on psoriasis: in a recent study, the intervention group was seemingly less likely to have a psoriasis flare than the control group over a 3 -year period (88 vs. $34 \% ; P<0.0001$ ) [21]. Taken together, these results suggest a role for physical exercise and, more generally, for lifestyle change in the management of skin psoriasis.

\section{PHYSICAL THERAPY, EXERCISE AND REHABILITATION: PERIPHERAL ARTHRITIS, DACTYLILTS, AXIAL INVOLVEMENT, FUNCTION AND QUALITY OF LIFE}

The majority of patients with PsA also have peripheral arthritis involvement, which contributes to decreased articular function and quality of life through the presence of both 
erosive and osteo-proliferative bone damage [22]. Conventional, targeted and biological disease-modifying anti-rheumatic drugs (DMARDs) have changed the management of articular involvement in PsA, although there are still patients in which articular damage evolves into limitations of function and quality of life [1]. In this context, physical therapy should be useful.

Physical therapy in the context of management of PsA should focus on different aspects of a patient's health, including general cardiovascular conditioning, strengthening exercises, improvement of articular motion and education. It may also provide modalities to decrease pain. Thermotherapy, which includes cryotherapy and heat, may be used to treat joint pain and to reduce swelling and tenderness (using cold) in inflamed joints. Paraffin baths are usually useful to reduce pain in hands and feet. Finally, orthosis and splinting are useful to prevent or reduce articular deformity [23].

In addtion of physical therapy, exercise is a key life-long strategy for the management of people with inflammatory arthritis and is recommended in clinical guidelines to manage symptoms, disability and comorbidity. Recent European League Against Rheumatism (EULAR) guidelines include recommendations for physical activity for people with inflammatory arthritis, including PsA [24]. General physical activity recommendations include four domains (cardiorespiratory fitness, muscle strength, flexibility and neuromotor performance) and are applicable (feasible and safe) to people with inflammatory arthritis, including PsA. For those with PsA, the Canadian Spondylitis Association also recommends exercise, including physical exercise $(\geq 150 \mathrm{~min}$ of moderate intensity aerobic physical activity/ week), muscle-strengthening exercise, stretching exercise, heat or cold applications and kinesiotherapy, to maintain range of motion [25].

A recent systematic review evaluated the role of cardiorespiratory and strength exercise on disease activity in patients with inflammatory diseases and found high-to-moderate quality evidence for a small beneficial effect on disease activity scores and joint damage [26]. The authors also reported moderate-quality evidence for a small beneficial effect on erythrocyte sedimentation rate and on articular symptoms [26].

Taken together, these studies suggest a potential role of exercise and physical therapy in the treatment of inflammation and in the overall management of patients. However, most of the evidence comes from studies on rheumatoid arthritis (RA), with a surprising low number of studies involving patients with PsA despite a similar overall prevalence (and, to a certain extent, clinical presentation) of the two conditions.

A proposal for a rehabilitation program for patients with PsA was previously published [27] and includes muscle-strengthening exercise, general fitness, stretching exercises, physical therapy, occupational therapy and patient education (Table 1 ).

While there is an abundance of evidence from randomized controlled trials (RCTs) on inflammatory arthritis (including RA), some studies have recently assessed the role of exercise and physical therapy on the management of peripheral joint involvement in PsA. Thomsen et al. performed a clinical trial aimed to evaluate the impact of high-intensity interval training (HIIT) on disease activity and disease perception in patients with PsA and to evaluate whether a potential effect could be sustained for a longer period of time [28]. The exercise intervention was performed as a supervised HIIT workout, starting with a 10-min warm-up period, followed by $4 \times 4 \mathrm{~min}$ of exercise at $85-95 \%$ of the maximum heart rate with each 4 min period interrupted by $3 \mathrm{~min}$ of exercise at $70 \%$ of the maximum heart rate. Although no clear benefits of the HIIT on disease activity and pain were demonstrated, fatigue improved significantly and there was no decline in most of the outcome variables from baseline to 9 months, suggesting that physical activity (even hight intensity) is safe [28].

Roger-Silva et al. recently evaluated the efficacy of a resistance exercise program in a RCT involving patients with PsA. This is the first study to carry out a program of resistance exercises in patients with PsA [29]. The participants in the RCT performed resistance exercises 
Table 1 A proposal for physical therapy, exercise and rehabilitation for articular involvement in patients with psoriatic arthritis

\begin{tabular}{lll}
\hline Peripheral disease & Axial involvement & References \\
\hline General fitness and cardiorespiratory exercises & General fitness and cardiorespiratory exercises & {$[25-27]$} \\
Muscle-strengthening exercises & Muscle-strengthening exercises & {$[25-27]$} \\
Flexibility exercise & Flexibility exercise & {$[25]$} \\
Neuromotor performance & Neuromotor performance & {$[25]$} \\
Physical therapy & Physical therapy & {$[34]$} \\
Occupational therapy and Education & Occupational therapy and education & {$[34]$} \\
Balneotharapy and hydrotherapy & Postural exercise & {$[30,34,35]$} \\
& Breathing exercise & {$[34-36]$} \\
& Balneotherapy and hydrotherapy & {$[34,35]$} \\
\hline
\end{tabular}

for the upper limbs, lower limbs and trunk. The exercises were divided in three sets of 12 repetitions for each muscle group and were performed twice a week for 12 weeks. The intensity of the exercises was $60 \%$ of one maximum repetition and the rest interval between exercises was 1-2 min. The study showed that resistance exercises are effective in improving functional capacity, axial disease activity and the general quality of life, with an additional beneficial effect on pain. However, these improvements were not coupled to improved muscle strength, and there was no reduction of peripheral disease activity as measured by the Disease Activity Score-28 (DAS-28) [29].

Hydrotherapy may be used for PsA patients. Benefits have been reported for physical function, energy, sleep, cognitive function, ability to work and participation in the activities of daily living [30].

Finally, there is some evidence supporting the beneficial effects of balneotherapy in the treatment of peripheral involvement of PsA [31].

Dactylitis can be treated with a physical therapy approach, following the recommendations available for RA. The physical therapist can help the patient to maintain function and range of movement of the small joints affected. Patients may learn new, less painful ways of carrying out daily activities. In terms of joint involvement, thermal modalities may be used to provide pain and swelling relieves, in particular for more recalcitrant forms of chronic dactylitis resistant to pharmacological therapy [23]. However, no formal studies have been published.

Axial disease in PsA (axPsA) shows some similarities and differences with ankylosing spondylitis (AS). In PsA, the involvement of the spine could lead to functional impairment and chronic pain. In contrast, axPsA is generally characterized by a higher asymmetry of and less severe change to the spine, lower frequency of inflammatory back pain and older age at presentation [32, 33].

The evidence for physical therapy interventions and exercise in the management of axPsA mainly comes from studies on AS and axial spondyloarthritis (SpA) that have reported strong evidence for a beneficial role of exercise and activity [34]. In this context, Nash et al. proposed, after a systematic review of the literature, a treatment algorithm in which physiotherapy was the second-line therapy in axial PsA [35]. The cornerstone of physical therapy in axial SpA is regular exercise and, in a broader view, this concept could also be valid for the axPsA. Regular exercise has been shown to reduce disease activity, pain and stiffness and 
improve physical functioning, chest expansion, spinal mobility and cardiorespiratory performance in patients; it also has the potential to reduce depressive symptoms [36].

In patients with axSpA, exercise has shown to be an important part of the disease management, with a tendency to be more effective when done in the framework of a supervised outpatient group [37-39]. Evidence of its efficacy and effectiveness is strong, mainly when associated with pharmacological treatment. Trials have consistently demonstrated the beneficial effect of a combination of anti-tumor necrosis factor (TNF) therapy and an AS-specific exercise program, compared with either antiTNF treatment or exercise alone, for both selfreported measures (such as function and disease activity) and objective measures, such as the Bath Ankylosing Spondylitis Metrology Index $[40,41]$. Furthermore, the Assessment of SpondyloArthritis International Society (ASAS)/ EULAR recommendations clearly identified exercise as a key component of axSpA management [42]. This evidence may be also applied to patients with axPsA, in which the burden of disease is similar.

\section{PHYSICAL THERAPY, EXERCISE AND REHABILITATION: ENTHESITIS}

Enthesitis is the hallmark of SpA and could be present in 35-50\% of PsA patients [43]. Recent studies on mouse models suggest a potential role for biomechanical stress in the pathogenesis of enthesitis and synovitis [44]. Thus, beyond the pharmacological treatment, the management of enthesitis in patients with PsA through physical therapy and rehabilitation represents still an unmet need; to date, no highquality studies have focused on the role of physical therapy and rehabilitation or the possibility of a harmful effect on entheseal inflammation [45]. Also, despite the large-scale implementation of physical therapy, few studies have assessed the efficacy and effectiveness of physical therapy in the reduction of entheseal inflammation and in the improvement of function and quality of life in patients with PsA with active enthesitis.
Various different methods can be prescribed to treat enthesopathy of mechanical/degenerative origin, most of which focus on the Achilles tendon or lateral epicondyle enthesitis. In this context, home exercise programs should include $10 \mathrm{~min}$ of muscle-stretching exercise (2-3 times/day) for the calf. To minimize stress at the Achille's tendon level with weight bearing, exercise involving active motion of the foot and ankle, through the entire range, should be performed for about $1 \mathrm{~min}$ when patients have been resting for a long period and before getting up [46].

Physiotherapy programs should be performed taking into account the patient's specific presentation, clinical history and biomechanics. With this approach, physiotherapy may lead to most effective outcomes when combined with exercise. However, published evidence for these complex interventions is still lacking, particularly for longer-term outcomes [47]. One published trial assessed the short-term effect of physiotherapy by using different techniques, including application of ice, transverse friction massage, therapeutic ultrasound and exercise (concentric, eccentric, balance and proprioceptive drills) in patients with Achilles tendinopathy over a 4-week follow-up. A control group without any treatment was also enrolled. The results showed significant improvements in pain related to activity in the physiotherapy group compared with the controls [48].

In comparison, night splints and heel braces seem to provide no further benefits $[49,50]$. For more recalcitrant forms of Achilles tendon enthesopathy, the use of extracorporeal pulse activation therapy (EPAT) should be considered. This technique is based on low-frequency pulse waves delivered locally using a handheld applicator. The pulsed pressure wave is a safe, non-invasive method and has the effect of stimulating metabolism and blood circulation. The action of EPAT may also be useful to regenerate damaged tissue and to accelerate healing [51].

Low-level laser therapy (LLLT) and extracorporeal shock wave therapy (ESWT) could also be used for the treatment of enthesitis. The basic mechanism of LLLT is tissue stimulation and a 
resulting increase in the self-repair activity of living tissue. LLLT also increases the permeability of the cell membrane and enhances fibroblast activity by increasing cell metabolism. LLLT has been considered, in a pilot study, for the treatment of enthesitis in patients with juvenile SpA and found to be efficacious in terms of pain management, although strong evidence is still lacking [52]. The mechanism of action of ESWT is complex and is based on the release of growth factors associated with angiogenesis in the target tissue. ESWT may also promote tissue healing by increasing the formation of new vessels [53]. This therapy may be effective in reducing pain, inflammation and edema and altering enthesophyte morphology in patients with calcaneal enthesopathy, as shown in different studies $[53,54]$.

In a recent study, both LLLT and ESWT were assessed as a treatment for enthesitis in patients with SpA. Alpturker et al. recently demonstrated a significantly reduction of both plantar fascia thickness and pain perception, using both strategies, in patients with plantar fasciitis due to SpA, suggesting a potential role for LLLT and ESWT in the treatment of this clinical manifestation [55].

However, despite available evidence, very few studies have focused on the treatment of enthesitis in PsA specifically, and in SpA in general, using physical therapy and exercise. Thus, no formal recommendations are as yet available on the type, intensity and duration of physical therapy required for the treatment of enthesitis in PsA. This lack of recommendations represent an unmet need, even in the light of different pathogenetic events recently demonstrated in SpA.

\section{PHYSICAL THERAPY, EXERCISE AND REHABILITATION: COMORBIDITIES}

Psoriatic arthritis is frequently associated with different comorbidities [2]. Recent evidence has demonstrated an increased prevalence of cardiovascular diseases, mainly linked to the presence of chronic inflammation. A recent metaanalysis showed an increased risk (43\%) of cardiovascular diseases in patients with PsA, with an overall morbidity risk for myocardial infarction, cerebrovascular diseases and heart failure of 68,22 and $31 \%$, respectively, compared with the general population [56]. Risk factors for cardiovascular diseases (hypertension, diabetes mellitus, hyperlipidemia and obesity) are also increased in patients with PsA compared to those affected by RA and psoriasis alone [57]. Finally, metabolic syndrome (MetS) is quite common in patients with PsA and represents a challenge in the management these patients [2].

Adiposity and higher BMI are also associated with an increased risk of developing PsA.

Of interest, in the recent HUNT study, a population-based longitudinal survey conducted in Norway with $>35,000$ participants, compared with the highest level of physical activity, lower levels were associated with a slightly higher risk of PsA. Moreover, physical activity at high levels seemed to modify the risk of PsA in overweight/obese individuals [58]. Several studies have evaluated the effect of exercise on MetS, reporting a significant increase in aerobic and muscle fitness, lean mass, high-density lipoprotein levels and a reduction of abdominal fat [59]. Together with the evidence of a reduction of risk of PsA in overweight individuals, exercise may provide benefits for those patients with MetS and psoriasis.

In this context, beyond the pharmacological treatment, exercise programs may play an important role in lowering not only the cardiovascular risk in PsA, but even the risk to develop the disease. A recent review showed how exercise and dietary programs improve waist circumference, blood pressure, triglyceride level and fasting glucose in subjects with MetS [60]. In addition, a recent study showed how weight loss could be associated with the improvement of disease activity in patients with PsA [61]. Thus, as stated by the EULAR recommendations, physical activity is useful not only to reduce the burden of disease but even to treat cardiometabolic comorbidities.

Finally, fibromyalgia may be present in $9-17 \%$ of patients with PsA [2]. Although no formal studies have yet assessed the 
improvement of fibromyalgia symptoms in PsA patients who participate in physical therapy and exercise programs, there is some evidence that these approaches could improve musculoskeletal symptoms related to fibromyalgia. Furthermore, there is some evidence for the role of balneotherapy at the Dead Sea area, which appears to produce a statistically significant substantial improvement in the number of active joints and tender points in both male and female patients with fibromyalgia and PsA [62]. In this context, further studies are needed.

\section{CONCLUSION}

Current evidence has demonstrated the potential beneficial effects of physical therapy, exercise and rehabilitation in patients with PsA. However, data from the literature are scarce and, for axial involvement, mainly come from clinical experience in AS. Nevertheless, patients with active PsA with impaired function and quality of life or with comorbidities such as obesity, cardiovascular diseases or fibromyalgia may achieve further benefits from a non-pharmacological approach. Expanding traditional programs of exercises to include aerobic components, cardiorespiratory exercises and educational programs may improve clinical outcomes in all disease domains, although there are as yet no standardized protocols.

In this context, further studies based on clinical trials and rigorous methodology are needed to evaluate the efficacy of exercise and rehabilitation programs specifically for patients with this multifaceted disease.

\section{ACKNOWLEDGEMENTS}

Funding. No funding or sponsorship was received for this study or for the publication of this article.

Authorship. All named authors meet the International Committee of Medical Journal Editors (ICMJE) criteria for authorship for this article, take responsibility for the integrity of the work as a whole, and have given their approval for this version to be published.

Disclosures. Fabio Massimo Perrotta, Silvia Scriffignano, Devis Benfaremo, Mario Ronga, Michele Maria Luchetti and Ennio Lubrano declare they have nothing to disclose.

Compliance with Ethics Guidelines. This article is based on previously conducted studies and does not contain any studies with human participants or animals performed by any of the authors.

Data Availability. Data sharing is not applicable to this article as no datasets were generated or analyzed during the current study.

Open Access. This article is licensed under a Creative Commons Attribution-NonCommercial 4.0 International License, which permits any non-commercial use, sharing, adaptation, distribution and reproduction in any medium or format, as long as you give appropriate credit to the original author(s) and the source, provide a link to the Creative Commons licence, and indicate if changes were made. The images or other third party material in this article are included in the article's Creative Commons licence, unless indicated otherwise in a credit line to the material. If material is not included in the article's Creative Commons licence and your intended use is not permitted by statutory regulation or exceeds the permitted use, you will need to obtain permission directly from the copyright holder. To view a copy of this licence, visit http://creativecommons.org/licenses/by$\mathrm{nc} / 4.0 /$.

\section{REFERENCES}

1. Ritchlin CT, Colbert RA, Gladman DD. Psoriatic arthritis. N Engl J Med. 2017;376(10):957-70. https://doi.org/10.1056/NEJMra1505557.

2. Lubrano E, Scriffignano S, Perrotta FM. Multimorbidity and comorbidity in psoriatic arthritis-a perspective. Expert Rev ClinImmunol. 2020. https://doi.org/10.1080/1744666X.2021.1825941. 
3. Lubrano E, Perrotta FM, Scriffignano S, Coates LC, Helliwell P. Sustained very low disease activity and remission in psoriatic arthritis patients. Rheumatol Ther. 2019;6(4):521-8. https://doi.org/10.1007/ s40744-019-00171-w.

4. Smolen JS, Schöls M, Braun J, et al. Treating axial spondyloarthritis and peripheral spondyloarthritis, especially psoriatic arthritis, to target: 2017 update of recommendations by an international task force. Ann Rheum Dis. 2018;77(1):3-17. https://doi.org/ 10.1136/annrheumdis-2017-211734.

5. Toussi A, Maverakis N, Le ST, Sarkar S, Raychaudhuri SK, Raychaudhuri SP. Updated therapies for the management of psoriatic arthritis. ClinImmunol. 2020;220:108536. https://doi.org/10.1016/ j.clim.2020.108536.

6. Lubrano E, Parsons WJ, Perrotta FM. Assessment of response to treatment, remission, and minimal disease activity in axial psoriatic arthritis treated with tumor necrosis factor inhibitors. J Rheumatol. 2016;43(5):918-23. https://doi.org/10.3899/ jrheum.151404.

7. Perrotta FM, Marchesoni A, Lubrano E. Minimal disease activity and remission in psoriatic arthritis patients treated with anti-TNF- $\alpha$ drugs. J Rheumatol. 2016;43(2):350-5.

8. Hagège B, Tan E, Gayraud M, Fautrel B, Gossec L, Mitrovic S. Remission and low disease activity in psoriatic arthritis publications: a systematic literature review with meta-analysis. Rheumatology. 2020;59(8):1818-25. https://doi.org/10.1093/ rheumatology/keaa030.

9. Mease PJ, Lertratanakul A, Anderson JK, et al. Upadacitinib for psoriatic arthritis refractory to biologics: SELECT-PsA 2. Ann Rheum Dis. 2020. https://doi.org/10.1136/annrheumdis-2020218870 .

10. Lubrano E, Scriffignano S, Perrotta FM. Residual disease activity and associated factors in psoriatic arthritis. J Rheumatol. 2020;47(10):1490-5.

11. Orbai AM, de Wit M, Mease PJ, et al. Updating the psoriatic arthritis (PsA) core domain set: a report from the PsA workshop at OMERACT 2016. J Rheumatol. 2017;44:1522-8.

12. Gasparyan AY, Ayvazyan L, Blackmore H, et al. Writing a narrative biomedical review: considerations for authors, peer reviewers, and editors. Rheumatol Int. 2011;31:1409-17.

13. Paroutoglou K, Papadavid E, Christodoulatos GS, Dalamaga M. Deciphering the association between psoriasis and obesity: current evidence and treatment considerations. Curr Obes Rep. 2020;9(3):165-78.

14. Fortes C, Mastroeni S, Leffondré K, et al. Relationship between smoking and the clinical severity of psoriasis. Arch Dermatol. 2005;141(12):1580-4.

15. Stewart TJ, Tong W, Whitfeld MJ. The associations between psychological stress and psoriasis: a systematic review. Int $\mathrm{J}$ Dermatol. 2018;57(11): 1275-82.

16. Rousset L, Halioua B. Stress and psoriasis. Int J Dermatol. 2018;57(10):1165-72.

17. Murray ML, Bergstresser PR, Adams-Huet B, Cohen JB. Relationship of psoriasis severity to obesity using same-gender siblings as controls for obesity. Clin Exp Dermatol. 2009;34(2):140-4.

18. Alotaibi HA. Effects of weight loss on psoriasis: a review of clinical trials. Cureus. 2018;10(10):e3491.

19. Ko SH, Chi CC, Yeh ML, Wang SH, Tsai YS, Hsu MY. Lifestyle changes for treating psoriasis. Cochrane Database Syst Rev. 2019;7(7):CD011972. https:// doi.org/10.1002/14651858.CD011972.pub2.

20. Naldi L, Conti A, Cazzaniga S, et al. Diet and physical exercise in psoriasis: a randomized controlled trial. Br J Dermatol. 2014;170(3):634-42. https://doi.org/10.1111/bjd.12735.

21. Li YF, Jiang LJ, Tao Y, Qin GL, Li Z, He W. The effects of walking exercise combined with continuous health education on prevention of psoriasis recurrence. J Nurs Adm. 2015;15(4):294-6.

22. Mahmood F, Coates LC, Helliwell PS. Current concepts and unmet needs in psoriatic arthritis. Clin Rheumatol. 2018;37(2):297-305.

23. Kavuncu V, Evcik D. Physiotherapy in rheumatoid arthritis. Med Gen Med. 2004;6(2):3.

24. Rausch Osthoff AK, Niedermann K, Braun J, et al. 2018 EULAR recommendations for physical activity in people with inflammatory arthritis and osteoarthritis. Ann Rheum Dis. 2018;77(9): 1251-60. https://doi.org/10.1136/annrheumdis2018-213585.

25. Canadian Spondylitis Association. https:// spondylitis.ca/wp-content/uploads/2018/10/2018PSA.

26. Sveaas SH, Smedslund G, Hagen KB, et al. Effect of cardiorespiratory and strength exercises on disease activity in patients with inflammatory rheumatic diseases: a systematic review and meta-analysis. Br J Sports Med. 2017;51:1065-72. 
27. Lubrano E, Spadaro A, Parsons WJ, Atteno M, Ferrara N. Rehabilitation in psoriatic arthritis. J Rheumatol Suppl. 2009;83:81-2.

28. Thomsen RS, Nilsen TIL, Haugeberg G, Bye A, Kavanaugh A, Hoff M. Impact of high-intensity interval training on disease activity and disease in patients with psoriatic arthritis: a randomized controlled trial. Arthritis Care Res. 2019;71(4): 530-7. https://doi.org/10.1002/acr.23614.

29. Roger-Silva D, Natour J, Moreira E, Jennings F. A resistance exercise program improves functional capacity of patients with psoriatic arthritis: a randomized controlled trial. Clin Rheumatol. 2018;37(2):389-95. https://doi.org/10.1007/ s10067-017-3917-x.

30. Lindqvist M, Gard G. Hydrotherapy treatment for patients with psoriatic arthritis-a qualitative study. Open J TherRehabil. 2013;1:22-30. https:// doi.org/10.4236/ojtr.2013.12005.

31. Cozzi F, Ciprian L, Carrara M, et al. Balneotherapy in chronic inflammatory rheumatic diseases-a narrative review. Int J Biometeorol. 2018;62(12): 2065-71. https://doi.org/10.1007/s00484-018$1618-\mathrm{z}$

32. Feld J, Chandran V, Haroon N, Inman R, Gladman D. Axial disease in psoriatic arthritis and ankylosing spondylitis: a critical comparison. Nat Rev Rheumatol. 2018;14(6):363-71.

33. Helliwell PS. Axial disease in psoriatic arthritis. Rheumatology. 2020;59(6):1193-5.

34. Perrotta FM, Musto A, Lubrano E. New insights in physical therapy and rehabilitation in axial spondyloarthritis: a review. Rheumatol Ther. 2019;6(4):479-86. https://doi.org/10.1007/s40744019-00170-х.

35. Nash P. Therapies for axial disease in psoriatic arthritis. A systematic review. J Rheumatol. 2006;33:1431-4.

36. Millner JR, Barron JS, Beinke KM, et al. Exercise for ankylosing spondylitis: an evidence-based consensus statement. Semin Arthritis Rheum. 2016;45: 411-27. https://doi.org/10.1016/j.semarthrit.2015. 08.003 .

37. Regel A, Sepriano A, Baraliakos X, et al. Efficacy and safety of non-pharmacological and non-biological pharmacological treatment: a systematic literature review informing the 2016 update of the ASAS/ EULAR recommendations for the management of axial spondyloarthritis. RMD Open. 2017;3: e000397.
38. Hilberdink B, van der Giesen F, Vliet Vlieland T, et al. Supervised group exercise in axial spondyloarthritis: patients' satisfaction and perspective on evidence-based enhancements. Arthritis Care Res. 2019;1:1.

39. O'Dwyer T, O'Shea F, Wilson F. Exercise therapy for spondyloarthritis: a systematic review. Rheumatol Int. 2014;34:887-902.

40. Lubrano E, D'Angelo S, Parsons WJ, et al. Effects of a combination treatment of an intensive rehabilitation program and etanercept in patients with ankylosing spondylitis: a pilot study. J Rheumatol. 2006;33:2029-34.

41. Lubrano E, Spadaro A, Amato G, et al. Tumour necrosis factor alpha inhibitor therapy and rehabilitation for the treatment of ankylosing spondylitis: a systematic review. Semin Arthritis Rheum. 2015;44:542-50.

42. van der Heijde D, Ramiro S, Landewé R, et al. 2016 update of the ASAS-EULAR management recommendations for axial spondyloarthritis. Ann Rheum Dis. 2017;76:978-91.

43. Kaeley GS, Eder L, Aydin SZ, Gutierrez M, Bakewell C. Enthesitis: a hallmark of psoriatic arthritis. Semin Arthritis Rheum. 2018;48(1):35-43. https:// doi.org/10.1016/j.semarthrit.2017.12.008.

44. Gracey E, Burssens A, Cambré I, et al. Tendon and ligament mechanical loading in the pathogenesis of inflammatory arthritis. Nat Rev Rheumatol. 2020;16(4):193-207. https://doi.org/10.1038/ s41584-019-0364-X.

45. Perrotta FM, Lories R, Lubrano E. To move or not to move: the paradoxical effect of physical exercise in axial spondyloarthritis. RMD Open. 2021;7(1): e001480. https://doi.org/10.1136/rmdopen-2020001480 .

46. Alfredson $\mathrm{H}$, Pietila $\mathrm{T}$, Jonsson $\mathrm{P}$, Lorentzon R. Heavy-load eccentric calf muscle training for the treatment of chronic Achilles tendinosis. Am J Sports Med. 1998;26(3):360-6.

47. Scott A, Huisman E, Khan K. Conservative treatment of chronic Achilles tendinopathy. CMAJ. 2011;183(10):1159-65.

48. Mayer F, Hirschmuller A, Muller S, et al. Effects of short-term treatment strategies over 4 weeks in Achilles tendinopathy. Br J Sports Med. 2007;41:e6.

49. Petersen W, Welp R, Rosenbaum D. Chronic Achilles tendinopathy: a prospective randomized study comparing the therapeutic effect of eccentric training, the AirHeel brace, and a combination of both. Am J Sports Med. 2007;35:1659-67. 
50. de Vos RJ, Weir A, Visser RJ, et al. The additional value of a night splint to eccentric exercises in chronic midportion Achilles tendinopathy: a randomised controlled trial. Br J Sports Med. 2007;41: e5.

51. Ordahan B, Türkoğlu G, Karahan AY, Akkurt HE. Extracorporeal shockwave therapy versus kinesiology taping in the management of plantar fasciitis: a randomized clinical trial. Arch Rheumatol. 2017;32(3):227-33.

52. Harjacek M, Kelava T, Lamot L. The therapeutic value of low-energy laser (LLLT) for enthesitis in children with juvenile spondyloarthropathies. Pediatr Rheumatol Online J. 2008;6(Suppl 1): P64-P64.

53. Cosentino R, Falsetti P, Manca S, et al. Efficacy of extracorporeal shock wave treatment in calcaneal enthesophytosis. Ann Rheum Dis. 2001;60:1064-7.

54. Aqil A, Siddiqui MR, Solan M, et al. Extracorporeal shock wave therapy is effective in treating chronic plantar fasciitis: a meta-analysis of RCTs. Clin Orthop Relat Res. 2013;471:3645-52.

55. ArmaganAlpturker K, Cerrahoglu ABL, Orguc IS. Evaluation effects of laser therapy and extracorporeal shock wave therapy with clinical parameters and magnetic resonance imaging for treatment of plantar fasciitis in patients with spondyloarthritis: a randomized controlled trial. Int $\mathrm{J}$ Rheumatol. 2020;2020:4386361.

56. Polachek A, Touma Z, Anderson M, et al. Risk of cardiovascular morbidity in patients with psoriatic arthritis: a meta-analysis of observational studies. Arthritis Care Res. 2017;69(67):74.
57. Radner $\mathrm{H}$, Lesperance $\mathrm{T}$, Accortt $\mathrm{NA}$, et al. Incidence and prevalence of cardiovascular risk factors among patients with rheumatoid arthritis, psoriasis, or psoriatic arthritis. Arthritis Care Res. 2017;69: 1510-8.

58. Thomsen RS, Nilsen TI, Haugeberg G, Gulati AM, Kavanaugh A, Hoff M. Adiposity and physical activity as risk factors for developing psoriatic arthritis. Longitudinal data from the HUNT study. Arthritis Care Res (Hoboken). 2021;73(3):432-41. https://doi.org/10.1002/acr.24121.

59. Myers J, Kokkinos P, Nyelin E. Physical activity, cardiorespiratory fitness, and the metabolic syndrome. Nutrients. 2019;11(7):1652. https://doi.org/ $10.3390 /$ nu11071652.

60. Konerman MA, Walden P, Joseph M, Jackson EA, Lok AS, Rubenfire M. Impact of a structured lifestyle programme on patients with metabolic syndrome complicated by non-alcoholic fatty liver disease. Aliment Pharmacol Ther. 2019;49(3):296-307.

61. Klingberg E, Björkman S, Eliasson B, Larsson I, Bilberg A. Weight loss is associated with sustained improvement of disease activity and cardiovascular risk factors in patients with psoriatic arthritis and obesity: a prospective intervention study with two years of follow-up. Arthritis Res Ther. 2020;22(1): 254.

62. Sukenik S, Baradin R, Codish S, et al. Balneotherapy at the Dead Sea area for patients with psoriatic arthritis and concomitant fibromyalgia. Isr Med Assoc J. 2001;3(2):147-50. 